\title{
Éditorial
}

\section{La recherche au-delà de 1999}

Il s'est déjà écoulé presque un an depuis la clôture de l'Année internationale des personnes âgées (AIPA). L'AIPA nous a permis d'augmenter la sensibilisation au vieillissement de la population et au statut des ainés ${ }^{1}$ de différentes parties du monde. Le Canada a été l'hôte d'une conférence de la Fédération internationale du vieillissement qui a réuni à Montréal divers groupes du monde entier dont les intérêts portaient sur les politiques à l'égard des aînés et la défense de leurs droits. La conférence annuelle de l'Association canadienne de gérontologie à Ottawa a abordé les défis du prochain millénaire et a servi de tribune à la constitution d'une vision des politiques, des pratiques et des éléments de recherche nécessaires au traitement des besoins de la prochaine génération d'aînés canadiens. Le Conseil consultatif national sur le troisième âge (CCNTA) a profité de l'attention accordée au vieillissement pour produire un document reflétant leurs opinions sur des questions importantes dans ce domaine pour la décennie qui s'annonce (CCNTA, 1999). On y traite notamment de la diversité des besoins et des ressources des aînés, de la situation de l'économie et de la santé ainsi que des conditions de vie.

Dans le cadre des activités de l'AIPA, l'ACG a entrepris l'élaboration d'un programme de recherche sur le vieillissement en se fondant sur le document du CCNTA. Des représentants de différentes divisions de l'ACG ont amorcé le travail en rédigeant des documents sur des thèmes de recherche. On leur avait demandé d'envisager les éléments d'un programme national de recherche en fonction de leur propre discipline, soit en santé, en sciences biologiques, en politique sociale, en psychologie et en sciences sociales. Les documents ont ensuite été présentés dans le cadre d'un colloque de la conférence de l'ACG d'Ottawa.

Les points soulevés dans les documents se rapprochaient sensiblement les uns des autres. On y signalait entre autres le fait qu'un programme de recherche sur le vieillissement doit reposer sur une analyse systématique des contextes et des théories qui forment nos sujets de recherche; qu'il y a des thèmes principaux qui se recoupent entre les disciplines; qu'il faut adopter de nouvelles structures de recherches. Le présent éditorial résume notre perspective sur les trois principaux éléments d'une recherche nationale sur le vieillissement: les contextes, les thèmes et enfin les structures et les approches.

\section{Les contextes de la recherche}

Une conclusion importante du colloque traitait du besoin d'aborder les contextes idéologiques de la recherche en gérontologie. En tant qu'experts, 
les chercheurs ont le pouvoir indéniable de déterminer les thèmes du vieillissement qu'il convient d'examiner. Les questions que l'on se pose ou non forment la base des connaissances gérontologiques. La recherche axée sur la curiosité a constitué le moteur du milieu universitaire et a donné lieu à des secteurs de recherches très vastes, allant des changements normatifs et cognitifs reliés à l'âge aux relations familiales des aînés.

Et pourtant, les chercheurs ne sont pas à l'abri de l'influence des valeurs qui dominent au sein du public ni des priorités institutionnelles. Ainsi, si l'on examine attentivement le document du CCNTA (CCNTA, 1999), on cons-tate que les préoccupations de santé viennent en tête. Cette prédominance découle du fait que les ressources financières et humaines consacrées aux soins de santé sont vastes et évidentes et qu'elles font l'objet d'un débat public important. Cependant, on perçoit le pouvoir du secteur de la santé des dix dernières années dans la forme que prend le programme de recherche sur les aînés, soit elle d'un "problème de soins» et à la solution reposant sur les "soins familiaux" qui en découle.

Le discours du milieu universitaire sur l'orientation à prendre en matière de recherche sur la gérontologie doit traiter de la façon dont le programme de recherche influe sur le type de questions que l'on se pose, sur celles que l'on n'aborde pas et sur l'interprétation des résultats. Une approche repose sur l'analyse critique des politiques par laquelle on examine les valeurs et les hypothèses d'un domaine particulier. Une deuxième approche se fonde sur l'examen documentaire, surtout dans les secteurs préoccupants en matière de politiques. C'est un relevé qui peut profiter aux chercheurs et aux responsables des politiques en consolidant l'information, en clarifiant les directions à prendre et en faisant ressortir les valeurs inhérentes au travail examiné. À titre d'exemple, on peut citer le numéro spécial de La Revue canadienne du vieillissement (printemps 2000) consacré à l'examen systématique de la documentation sur l'autonomie des aînés. Ce genre d'efforts révèle à la fois la profondeur considérable des aspects du vieillissement qui nous sont connus et la limite de notre compréhension de leur création. Les faits ne parlent pas d'eux-mêmes. Comprendre leur origine nécessite des cadres théoriques solides qui font progresser leur explication.

\section{Les sujets de recherche}

Nous relevons ici trois principaux secteurs d'un programme de recherche sur le vieillissement. Ils ont été établis à partir des thèmes examinés dans le document du CCNTA et de notre évaluation collective des thèmes qui se recoupent dans les différentes disciplines. Nous les avons choisis parce qu'ils représentent de nouvelles perspectives sur des sujets principaux reliés au vieillissement de la personne et de la population et parce qu'ils entraînent une réévaluation à la lumière de l'influence des contextes de recherche actuels. Les secteurs de recherche sont le contexte familial du 
vieillissement, la gestion des états de maladies complexes et la qualité de la vie.

Les contextes familial et social du vieillissement

Les démographes et autres experts du domaine social ont étudié les changements substantiels de la structure familiale résultant du divorce et du remariage et les courbes de fertilité. Nous connaissons néanmoins très peu de choses sur la vie familiale des aînés canadiens. La recherche sur les questions de famille normative, notamment sur la construction sociale des aînés sur le plan des membres de la famille, est essentielle à notre compréhension de l'isolation, de la solitude et des variations de la composition des systèmes d'appui primaires. Dans le même ordre d'idées, la recherche sur la façon dont les familles abordent les transitions normatives du travail à la retraite et les conditions de logement en fin de vie sont essentielles au développement et au raffinement de la sécurité du revenu et des politiques de logement répondant aux besoins des familles âgées.

La prestation des soins quotidiens se produit en fin de vie chez les Canadiens et leurs familles. Ces derniers gèrent la prestation et la réception des soins et doivent conjuguer avec les autorités de soins structurés et avec leurs propres activités professionnelles et récréatives. Il est temps d'aller au-delà de l'étude des fournisseurs et des bénéficiaires de soins car c'est une approche qui limite notre compréhension de la capacité des familles à faire face aux nombreuses demandes. La prochaine étape consiste à étudier le développement et les activités des réseaux de soins non structurés au fur et à mesure que nous nous approchons de l'établissement de systèmes de soins communautaires et domestiques à l'égard des aînés qui en ont besoin. C'est un travail qui doit être mené dans une perspective d'examen rigoureux des hypothèses du secteur encore inexploré des soins non structurés. Dans le passé, le Canada a adopté une approche collective à la prestation des services sociaux. Les individus, les familles et les bénévoles ne peuvent remplacer les services publics.

La gérontologie clinique: la constatation et la gestion des maladies complexes

On a accompli des progrès importants en gérontologie clinique et en médecine gériatrique sur le plan de la gestion des maladies aiguës et chroniques reliées à la fragilité, de la santé mentale et de l'évaluation de la santé. II convient maintenant d'accorder la priorité à l'examen approfondi de l'étiologie et de la gestion de la déficience intellectuelle. Au coeur des préoccupations sur lesquelles repose l'hypothèse d'une "crise démographique," règnent l'augmentation prévisible et disproportionnée de la démence et des états de maladie complexes associés à la fragilité.

Le public semble fortement s'intéresser à l'application d'interventions technologiques de survie pour les aînés, qui autrement se portent bien, même à un âge très avancé. Par contre, lorsque les aînés éprouvent des difficultés multiples, complexes, médicales et sociales, et plus particulière- 
ment lorsque ces problèmes compromettent leur fonctionnement, on constate une intolérance importante de la part du public à l'égard de la fourniture de soins impératifs. L'intolérance s'exprime par l'utilisation de termes dénigrants comme "monopolisateur de lit,» "chroniques» et "admissions sociales" qui font maintenant partie du vocabulaire médiatique et du langage des politiques. Il faut établir de nouveaux modèles de soins facilement accessibles pour les patients dont l'état a atteint un degré de complexité avancé. Il faut également orienter la recherche vers le traitement de la défaillance multiorganique. C'est une erreur de tenter de réduire ces états de maladie complexes à la simple somme des déficiences individuelles des organes. C'est une erreur également de supposer que la recherche sur les états de maladie complexes associés au vieillissement ne doit être menée que par des organismes financés qui effectuent de la recherche sur des maladies particulières.

Nous devons développer de meilleurs outils pour déterminer les troubles de démence, par exemple, la maladie d'Alzheimer et les troubles de déficience intellectuelle sans démence. Un diagnostic précis en début de maladie augmente notre capacité de maximiser les effets des agents pharmaceutiques et des autres formes de traitement.

Il faut poursuivre la recherche de base en pathophysiologie de la démence et inclure ces études et leur application aux expérimentations sur les médicaments. Il faut développer et évaluer de meilleures approches comportementales en matière de soins des aînés souffrant de déficience intellectuelle et de troubles de comportement. Le contexte de cette forme de recherche doit prendre en compte la diversité familiale et ethnique en réaction à la gestion de la démence, la prestation des services en milieu rural à l'égard des déficients intellectuels et enfin, l'intégration des services et des secteurs sociaux de santé et de logement.

\section{La qualité de la vie}

Les changements sur le plan de la biologie, de la sensation, de la perception et de la cognition chez les aînés peuvent entraîner des effets profonds sur la qualité de leur vie. Nous avons accompli des progrès importants dans la compréhension du fonctionnement de base des changements normatifs ou non dans ce domaine et le travail doit se poursuivre. Cependant, la priorité de la recherche repose sur une meilleure compréhension des modérateurs importants de l'impact de ces changements sur la qualité de vie des aînés. On doit pouvoir compter sur le recoupement entre les disciplines puisque les modérateurs peuvent comprendre des facteurs de style de vie (habitudes d'alimentation et modes d'exercices), une maladie ou une invalidité concurrente, le contexte social (relations avec les amis et activités sociales importantes) et enfin les relations spatiales (différences rurales et urbaines quant à l'accès aux services).

$\mathrm{Au}$ centre de la priorité de cette recherche se situe un ensemble de questions philosophiques concernant la définition de la qualité de la vie. La recherche actuelle repose sur l'hypothèse qu'une bonne qualité de vie 
résulte de l'autonomie et de la maîtrise de ses décisions dans des domaines comme les conditions de logement, la réception des services et l'interaction avec la famille et les amis. La priorité de la recherche sur la qualité de la vie doit reposer sur des questions entourant les circonstances dans lesquelles l'autonomie constitue le meilleur modèle de qualité de vie et se faire en fonction des situations économiques et sociales diverses de notre société multiculturelle.

\section{Les structures et les approches de la recherche}

Les valeurs du public et les priorités institutionnelles influencent la façon dont la recherche est menée, tout comme le font les structures et les approches de la recherche. La récente formation des Instituts de recherche en santé du Canada (IRSC) et la constitution d'organismes de financement connexes auront une influence marquée sur la recherche. Par l'entremise des IRSC, le gouvernement fédéral entend appuyer les programmes collectifs de recherche sur la santé qui visent la création et la mise en pratique de connaissances sur les systèmes de santé. Le débat se poursuit sur les approches de recherche interdisciplinaire, sur la définition de la santé et sur l'équilibre entre la recherche fondée sur les politiques et celle qui repose sur l'enquête ou la curiosité.

Le mouvement vers un programme de recherche national et les stratégies de financement ouvre la voie à une approche de recherche sur le vieillissement qui a été jusqu'ici absente de la scène canadienne, soit le développement de données longitudinales. Notre connaissance du vieillissement repose presque exclusivement sur un groupe de gens dont les conditions économiques et historiques et la situation familiale et de santé s'équivalent. Nous savons très peu de choses sur la façon dont les groupes qui suivront feront l'expérience de la maladie reliée à l'âge comme la démence et comment leurs familles seront constituées. Nous ne savons pas à quel genre de soutien non structuré ils auront accès, quel type de logement ou de services leur seront accessibles ou acceptables, quels types de systèmes de soins de santé ou de soins à domicile répondront à leurs besoins, ni quel genre d'anomalies sociales ou économiques affectent le processus du vieillissement. La compréhension du processus nous oblige à mener une recherche longitudinale.

Le Canada dispose d'une certaine expertise en matière de santé et de caractère social du vieillissement. Des efforts collectifs pour concevoir une étude nationale longitudinale pourraient s'appuyer sur les divers secteurs d'expertise, permettre d'éviter le problème d'une généralisation restrictive reliée aux études de moindre envergure et aborder une vaste gamme de thèmes connexes. Il faut établir des données longitudinales qui s'étendront sur une période suffisamment longue (au moins 20 ans). Elles devront également couvrir une variété de recherche suffisamment vaste pour en faire une ressource importante pour les chercheurs des différentes disci- 
plines reliées à la gérontologie. À cet égard, on peut avantageusement s'inspirer du cycle d'enquêtes de Statistiques Canada en matière de santé et de questions sociales. À chaque programme de recherche rationnelle sur le vieillissement peuvent s'ajouter des recherches longitudinales moins élaborées.

Nous croyons qu'un programme canadien complet de recherche en gérontologie doit couvrir les trois secteurs présentés ici. Les priorités substantielles pourraient servir à faire avancer l'ensemble de nos connaissances. À titre d'exemples récents, on peut citer le Programme de recherche sur l'autonomie des aînés de même que l'Étude sur la santé et le vieillissement au Canada (dont l'accent est mis sur la démence) qui illustrent comment la collaboration entre chercheurs peut rehausser notre compréhension de questions complexes. Cependant, ces priorités doivent s'accompagner d'une critique de nos contextes et d'une élaboration de théories. Si nous ne voulons pas nous limiter à la constitution de données, il faut nous projeter au-delà de nos projets individuels vers une vision élargie de nos activités. Nous croyons que le temps est venu de nous engager dans une étude longitudinale nationale pour mieux examiner le processus du vieillissement.

\section{Notes}

1 Le masculin embrasse le féminin.

\section{Références}

Conseil consultatif national sur le troisième âge. (1999). 1999 and beyond: Challenges for an aging society. Ottawa: Minister of Public Works and Government Services Canada.

Ingrid Connidis, Norah Keating, Sheila Neysmith, Colin Powell, Kenneth Rockwood, et Mary Tierney 